DOI:

10.1038/nri1972

\section{Off the beaten path}

A new study in Nature Immunology has reassessed the developmental origins of thymic natural killer (NK) cells and describes an alternative pathway of NK-cell development in mice that is characterized by the expression of the zinc-finger transcription factor GATA3 (GATAbinding protein 3) and CD127 (the $\alpha$-chain of the interleukin-7 receptor).

NK cells are one of the main effector arms of the innate immune system. They are known to be generated in the bone marrow, but they are also found in the liver, the lymph nodes and the thymus. It is possible that, in different tissues, NK cells undergo local differentiation processes, but there is also evidence to indicate that there might be several NK-cellprecursor pools. Phenotypically and functionally distinct subsets of mature NK cells, however, have yet to be identified in adult mice. To

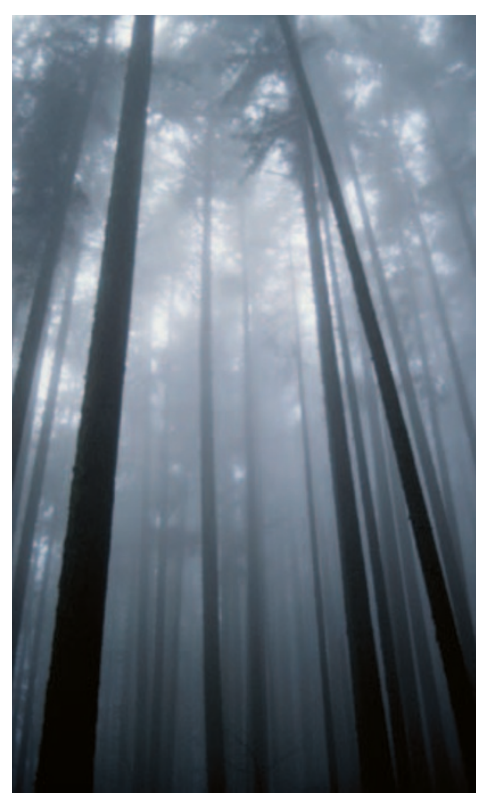

address this problem, James Di Santo and colleagues focused on the small population of NK cells that is present in the fetal and adult thymus.

GATA3 is required for the generation of early T-cell precursors in the thymus and has been shown to be involved in the differentiation of NK cells. The authors therefore compared the expression of GATA3 by NK cells from different anatomical sites. NK cells in the bone marrow and liver had low GATA3 expression, whereas those from the spleen had no GATA3 expression. All thymic NK cells, however, had high expression of GATA3, indicating that NK cells in the thymus differentiated in situ and that they differed from the pool of circulating peripheral NK cells. CD127, which might be a potential target of GATA3 expression in early double-negative thymocytes, was also expressed by most thymic NK cells but not by splenic NK cells. Additional characterization of cellsurface markers further distinguished thymic CD $127^{+}$NK cells from those in the spleen.

Do thymic CD $127^{+} \mathrm{NK}$ cells contribute to the peripheral NKcell pool? Two subsets of NK cells derived from the peripheral lymph nodes were identified - those with a CD $127^{+}$('thymic') phenotype and those with a CD127- ('splenic') phenotype - indicating that peripheral CD $127^{+} \mathrm{NK}$ cells might be derived from a thymic precursor. Indeed, CD $127^{+}$NK cells were subsequently shown to be exported from the thymus and to localize to peripheral lymphoid organs.

The generation of thymus-derived $\mathrm{CD} 127^{+} \mathrm{NK}$ cells was dependent on GATA3 expression and interleukin-7, unlike NK cells generated in the bone marrow. Functionally, compared with splenic CD127 ${ }^{-}$NK cells, thymic $\mathrm{CD} 127^{+} \mathrm{NK}$ cells showed reduced cytotoxic activity, possibly as a result of their relatively immature phenotype (CD11b ${ }^{\text {low }}$, with a poorly developed NK-cell-receptor repertoire), yet they were strong producers of cytokines. Together, these data indicate that $\mathrm{CD} 127^{+} \mathrm{NK}$ cells are in fact mature and are functionally distinct from splenic CD127- NK cells.

The two human NK-cell subsets (which are distinguished on the basis of their expression of CD16 and CD56) show similar phenotypic and functional differences. Di Santo and colleagues therefore asked whether the mouse NK-cell subsets distinguished by CD127 expression were analogous to the human NK-cell subsets. The human subsets did indeed differentially express both GATA3 and CD127, similar to the mouse subsets, indicating that the two human and the two mouse NK-cell subsets might have similar developmental and functional properties.

So the results of this study point to there being both bone-marrow and thymic pathways of NK-cell development in mice, resulting in the generation of distinct subsets of NK cells. More work will be needed to characterize fully the thymus-derived NK cells, and the authors suggest that the broad cytokine-expression profiles of these cells (and the possible plasticity of these profiles in different stimulatory environments) should be the focus of future studies.

Sharon Ahmad

ORIGINAL RESEARCH PAPER Vosshenrich, C. A. J. et al. A thymic pathway of mouse natural killer cell development characterized by expression of GATA-3 and CD127. Nature Immunol. 1 Oct 2006 (doi:10.1038/ni1395) 